\title{
Application of Fuzzy Clustering Methodology for Garment Sizing
}

\section{Adepeju Abimbola Opaleye*, Adekunle Kolawole, Oliver Ekepre Charles-Owaba}

Department of Industrial and Production Engineering, University of Ibadan, Ibadan, Nigeria

\section{Email address:}

aa.opaleye $a$ ui.edu.ng (A. A. Opaleye), k.kolawole@maill.ui.edu.ng (A. Kolawole), oe.charles-owaba@maill.ui.edu.ng (O. E. Charles-Owaba)

${ }^{*}$ Corresponding author

\section{To cite this article:}

Adepeju Abimbola Opaleye, Adekunle Kolawole, Oliver Ekepre Charles-Owaba. Application of Fuzzy Clustering Methodology for Garment Sizing. American Journal of Data Mining and Knowledge Discovery. Vol. 4, No. 1, 2019, pp. 24-31. doi: 10.11648/j.ajdmkd.20190401.15

Received: April 16, 2019; Accepted: May 28, 2019; Published: June 12, 2019

\begin{abstract}
With the growing demand for Ready-To-Wear outfits especially in African textile prints, the currently used European, American and Asian garment sizing systems seems unsuitable for the Nigerian garment industry where customer's choose clothing item not only due to fit in terms of body measurements but also the dress culture, style, preference and some other implicit requirements. This study aims to develop a size chart for different styles of trousers worn by Nigeria male population. Anthropometric data of 500 customers were taken in a natural random process and from stable tailoring establishments. The data was analysed using descriptive statistics and the fuzzy clustering methodology (FCM) was used as a suggestive approach which describes subjectivity in customer preferences. Analysis of the FCM output shows that the number of individual measurements with misfit has no significant difference $\left(\mathrm{F}_{\text {estimated }}=1.119, \mathrm{p}\right.$-value $=0.375$ and $\left.\mathrm{F}_{\text {critical }}=2.866\right)$ across cluster. The percentages of misfit were 38.0, 23.4, 31.6, 31.4 and 3.8\% for hip measurement, length, waist, thigh and bottomgirth respectively. The developed sizing system which reflects subjectivity in customer's selection of trouser may also enhance both producer and retailer's production and replenishment policy=
\end{abstract}

Keywords: Ready-To-Wear, Size Chart, Trousers, Fuzzy Clustering

\section{Introduction}

Variation in human body dimensions is a vital consideration in product and workplace design. This is also emphasized in the garment making and production industry, as customers purchase clothing items suitable to their body dimensions, dress culture and preferences [1]. There are ongoing efforts to establish anthropometric data-base for various populations. Accessibility to this data base makes it possible to design products suitable to a large percentage of a country population and enhance their perception on quality of locally produced garments.

However, in Nigeria, availability of locally made ReadyTo-Wear (RTW) garments is still in the growing stage. Most Nigerians acquire clothing items by visiting fashion designers/dressmakers or by the try-on method at boutique. This is due to the different sizing system used among domestic manufacturers and retailers. It is known that body dimensions and types differ with respect to ethnic diversity, age and gender of people in different countries [2]. Each country must have standard cloth sizing systems suitable to its dressing culture in order to guide production, purchases and facilitate rapid development of its RTW garment industry $[2,3]$.

With the growing demand for RTW outfits especially in African textile prints, the currently used European, American and Asian garment sizing systems seems unsuitable for most Nigerians [4]. Inherently, it becomes difficult for domestic designers to expand their customer baseand reduce production cost [3]. Therefore, the development of garment sizing system for Nigeria RTW designers, manufacturers, retailers and consumers is long overdue.

\section{Literature Review}

CS 215-58 Standard, an empirical sizing systems based on expert experience was first developed in USA in the year 1958. This was followed by the PS 42-70 Standard developed 
using military anthropometric data in 1970. The probably first scientific approach to garment sizing was achieved by Salusso-Deonier in 1982. Salusso [5] classified the anthropometric data of adult females in US. Using the principal component analysis, the researcher found 15 key components that summarize trends in body form variationIn a similar study, Tryfos [6] proposed the use of integer programming to determine optimal number of sizes required to minimize aggregate discomfort index. The body dimension taken was divided into a set of discrete possible sizes and the problem is formulated as a "Facility Location problem" so as to maximize expected sales. Another was by McCulloch et al. [7], thenon-linear optimization model was used to derive a set of possible sizing systems from US anthropometric data.

Recent attempts to establish standardized systems are prominent in studies from developing countries. Based on statistical data analysis, Gupta and Gangadhar [8] segregated 95 percent of the Indianpopulation into eleven size charts. They used a total of 21 anthropometric data for Indian women from six metro cities. Multivariate analysis was carried out to identify relationships between variables and principal component analysis was used to identify the key body measurements and create a basis for classifying the population. Gupta et al., [9] also considered the linear programming technique to derive a set of possible sizing system. Locker et al., [10] describe a variety of size-specific methods based on statistical and visual analysis which can be applied toimprove the apparel fit of a sizing system. This was applied to improve an existing sizing system of a garment production company. Lee [11] and Beshah et al., [12] examined the various sizing systems and specified anthropometric data as well as the fit issue in Korea using inferential statistics.

Saket and Pandya [13] proposed the clustering technique for exploring information from large quantities of data so as to understand pattern existing in the dataset. Partitioning clustering is a data mining approach useful in garment sizing. It is an unsupervised learning process of grouping similar data points. Itsmain objective is to divide the data points into ' $\mathrm{K}$ ' partitions. Zakaria et al., [14], Bagherzadeh et al., [15] and Elfaki and Ali [16] used principal component analysis to determine key anthropometric measurements and cluster analysis for the sizing system. Rao et al. [17] clustered 10096 anthropometric datasets of children in 54 districts of Uttar Pradesh into 4 clusters according to their average height and weight. Majumder and Sharma [18] classified homogenous human body size of 382 men and 391 women of Orissa in India using cluster analysis ofself reported age, stature, weight and percent body fat. Elfaki and Ali [16] also considered the K-Mean clustering technique for military clothing factory sizing chart.

In 2009, Hsu and Kuo [3] conducted a study on the development of size chart in order to improve business operations in Taiwan using the fuzzy clustering for analysis. Unlike, the crisp clustering which assigns data point (an individual) to only one cluster (size group), fuzzy clustering allows data points (individual) to belong to more than one group. The fuzzy clustering may be regarded as a suggestive approach which describes subjectivity in customer size preferences at different instances. In this case, customer choice of size is not only a function of measuredfit as \pm 1 body size but also that of design or style and preference [19, 20]. This is typical of African wear which comes in different forms and sizes. In addition, in many multi-item inventory environments such as the garment store or boutique, a joint replenishment policy is defined as the coordination of multiple cloth items that may be ordered jointly from a single producer [21]. Practically, a retailer is willing to buy more when the producer offers a price break or discount. Therefore, the fuzzy-based size chart may be used for translating different customer preferences to retailers and producers replenishment and production policies respectively.

The current study is therefore undertaken with the aim of developing an easy to use size chart for the design, production and sale of the different types of Nigeria male trousers.

\section{Overview of Fuzzy C-Means Clustering Methodology}

The Fuzzy C-Means clustering method was introduced as an extension of the Hard C-Mean clustering method [22]. It is an unsupervised clustering algorithm in which a data set is grouped into 'n' clusters. Each data point in the set is related to every cluster with a high degree of belonging to a particular cluster mid-point [23, 24]. The algorithm which minimises the distance between the a data point and cluster mid point is given as follows;

$$
Z=f\left(u, c_{1}, c_{2} \ldots c_{c}\right)=\sum_{i=1}^{C} \sum_{j=1}^{N} U_{i j}^{m}\left\|x_{j}-c_{i}\right\|^{2}
$$

Step 1: Assign the number of clusters, $C,(2<=i<C)$ to be considered and determine parameter ' $\mathrm{m}$ ' which is a weighting exponent on fuzzy membership.

Step 2: Initialize the membership matrix $U_{o}$ with random values between 0 and 1 such that the constraints in Equation (2) is satisfied.

$$
\sum_{i=1}^{C} U_{i j}=1, \nabla j=1,2, \ldots n
$$

Step 3: Compute fuzzy cluster $\operatorname{centers} c_{i}, \mathrm{i}=1, . ., \mathrm{C}$ using Equation (3)

$$
C_{i}=\frac{\sum_{j=1}^{n} U_{i j}^{m} x_{j}}{\sum_{j=1}^{n} U_{i j}^{m}}
$$

Step 4: Compute the objective function according to Equation (1).

Step 5: Compute a new membership matrix $U_{r}(\mathrm{r}=0,1,2 \ldots$ number of steps in the algorithm) using Equation (4).

$$
U_{i j}=\frac{1}{\sum_{k=1}^{C}\left[\left\|\frac{x_{j}-c_{i}}{x_{j}-c_{k}}\right\|\right]^{-2 /(m-1)}}
$$


Step 6: Go to step 3.

The iterations stop when the difference between the fuzzy partition matrices in two following iterations is lower than a specified $e$ [24].

\section{Methodology}

In this study, relevantanthropometric data for male trousers such as length, waist, thigh, hip and bottom girth were obtainedfroma sample of customers randomly taken instable fashion centres or establishments in fourcities in Nigeriawith large population of customers. This data obtained by Kolawole [25] were collected in Lagos, Ibadan, Kano and Jos. Data wereprocessed to separate out missing data. As a result, 500 samples of adult male's dimensions were valid for use. Sample is as shown in Appendix-

The process of data analysis involves three stages. The first is the process of data description. Data description allows the presentation of the collected data in a meaningful way which enhances interpretation. It involves the use of numerical descriptive measure and investigation of the pattern of statistical distribution evident in the collected data. The process of investigating pattern of statistical distribution observed in data sample of trousers taken from the tailors is achieved by calculating the sample statistics and relating them to the statistics of normal distribution using Kolmogorov-Smirnov (KS) test statistics. The 5\% critical KS values are given against the value of the test statistic. The test statistics is rejected if greater than the critical value [26]. This is achieved using the easyfit statistical software and Excel spreadsheet. The second stage involves clustering of collected data using the FCM clustering methodology as described in section 3.0 (The application is performed on NCSS 10 software package). The final stage is the process of validation using the average silhouette value and a measure of fit. The average silhouette value represents how closeness of observations within each cluster [3] while the measure of fitevaluate individualdimension to lower and upper border of the cluster dimension. A rating scale of good, decent or poor fit byHernández, et al., [27] was adapted in determining how well individual body dimension fit into a cluster.

$$
v_{i}=1-\left[\frac{\sqrt{ }\left(P_{1 j-G_{i j}}\right)}{P_{2 j-P_{1 j}}}\right]
$$

$v_{i}$ : Decent fit for individual garment measurement

$G_{i j}$ : Actual measure of individual $i$ for the dimension $j$.

$P_{1 j}, P_{2 j}$ : Lower and upper border of dimension $j$

$v_{i j}$ : Fit of individual $i$ measurement to dimension $j$

If one of the fit values was zero (0), the individual measurement is considered as total misfit for the cluster. This is so because if the individual dimensionhas a poor fit in any of the areas, then a good and/or decent fit in other areas cannot compensate for the poor fit and make the individual dimensionseem to fit the cluster. Further evaluation of the distribution of misfit was conducted using the analysis of variance (ANOVA) on Excel spreadsheet.

\section{Results and Discussion}

The descriptive statistics of the anthropometric variables for male trousers is presented in Table 1. In this study, individual men's basic trouser measurement was used $(\mathrm{n}=$ 500) and five measurements were selected: length, waist, thigh, hip and bottom-girth as critical dimensions. From Table 1, it is observed that the trouser length range from 93.98 to 119.38 (Mean $105.024 \mathrm{~cm}+4.709, \mathrm{n}=500$ ), the variable seem fairly skewed to the right (skewness $=0.279$ ), with a median of $104.14 \mathrm{~cm}$ and an inter-quartile range of 6.35. The 95th percentile, used as a definition of an extreme length value, was $114.3 \mathrm{~cm}$. Statistics for other dimension is as shown on Table 1. The result of the KS analysis with the test statistics greater than the critical values for all anthropometric attributes shown in Table 2 (KS test $=0.096$, $\mathrm{CV}<0.04$; visual examination of probability plot in Figure 1 to 5) affirms that none of the attribute datasets is normally distributed. In large datasets, this may not be out of place as there may be a mixture of two or more normal distributions. Also, given the data summary with the mean and standard deviation, if the interval (mean \pm 2 standard deviation) has infeasible limits, then this may indicate that any statistical tests with underlying normality assumption may be invalid. However, this is not the case with this dataset, the interval limits of all the attributes are feasible as such, and any statistical analysis is also applicable to the data set.

For the FCM based procedure, clustering of population data was carried on the basis of the five attributes (length, waist, hip, thigh and bottom-girth) and five clusters. The center value for each cluster per attribute is as shown on Table 3. This provides a guide for the customertrouser selection decision. To validate the quality of clusters formed, the average silhouette value for each of the specified number of cluster was obtained as shown in Figure 6. This represents how well observations were accrued within each cluster based on the selected base attribute or critical dimension. Average silhouette value ranges between -1 and +1 , and positive values indicate strong clustering, while negative values indicate weak clustering. A minimum of five (5) clusters is however considered in this study

To gain better insight into theclustersresulting from the five base attributes (length, waist, thigh, hip and bottom_girth) and identify misfit, the rating scale by Hernández, et al., [27] was used and the output of the FCM methodology analysed. Misfit of a trouser can be caused by a single measurement or by a combination of two or more measurements. However, a certain number of misfits is expected within any target group and for any clustering methodology. Based on the output on Table 4, 10.8\% $(n=500)$ of the individuals measurement clustered have one measurement judged as misfit (fit value $=0$ ). None of these individual measurements was found to have a misfit in more than one dimension. Further analysis showed that the number of individual measurements with misfit has no significant difference $\quad\left(\mathrm{F}_{\text {estimated }}=1.119, \quad \mathrm{p}\right.$-value $=0.375 \quad$ and Fcritical $=2.866$ ) across clusters using the $F$ test of ANOVA. 
The highest percentage of misfit of $38 \%$ was observed in waist, 31.4 for thigh and 3.8\% for the bottom-girth. relation to hip measurement, $23.4 \%$ for length, $31.6 \%$ for the

Table 1. Descriptive Statistics of anthropometric attributes.

\begin{tabular}{llllll}
\hline & Length & Waist & Thigh & Hip & Bottom_girth \\
\hline Count & 500 & 500 & 500 & 500 & 500 \\
Mean & 105.0239 & 82.25536 & 70.84314 & 96.94418 & 45.0342 \\
Standard Deviation & 4.708099 & 7.482402 & 3.821114 & 6.167234 & 3.490886 \\
Standard Error & 0.2105526 & 0.334623 & 0.170885 & 0.275807 & 0.1561172 \\
Lower 95\% CL Mean & 104.6102 & 81.59792 & 70.50739 & 96.40229 & 44.72747 \\
Upper 95\% CL Mean & 105.4376 & 82.9128 & 71.17889 & 97.48607 & 45.34093 \\
Median & 104.14 & 81.28 & 71.12 & 96.52 & 45.72 \\
Minimum & 93.98 & 68.58 & 58.42 & 68.58 & 38.1 \\
Maximum & 119.38 & 114.3 & 99.06 & 121.92 & 91.44 \\
Range & 25.4 & 45.72 & 40.64 & 53.34 & 53.34 \\
Interquartile Range & 6.35 & 8.89 & 5.08 & 5.08 & 2.54 \\
10th Percentile & 99.06 & 73.66 & 66.04 & 88.9 & 43.18 \\
25th Percentile & 101.6 & 77.47 & 68.58 & 93.98 & 43.18 \\
75th Percentile & 107.95 & 86.36 & 73.66 & 99.06 & 45.72 \\
95th Percentile & 114.3 & 96.52 & 77.47 & 109.22 & 48.28 \\
Variance & 22.1662 & 55.98634 & 14.60091 & 38.03477 & 12.18628 \\
Mean Absolute Deviation & 3.737133 & 5.799125 & 2.679842 & 4.554322 & 1.724558 \\
Mean Absolute Deviation from Median & 3.69824 & 5.62356 & 2.6289 & 4.50342 & 1.55448 \\
Coefficient of Variation & 0.0448288 & 0.090966 & 0.053938 & 0.063616 & 0.07751632 \\
Coefficient of Dispersion & 0.0355122 & 0.069188 & 0.036964 & 0.046658 & 0.034 \\
Skewness & 0.2788599 & 1.026052 & 1.138375 & 0.404719 & 8.077789 \\
Kurtosis & 2.993381 & 4.565249 & 10.11941 & 5.108311 & 102.9672 \\
\hline
\end{tabular}

Table 2. Kolmogorov-Smirnov Test Statistics for Trouser Data.

\begin{tabular}{llll}
\hline Attribute & Test statistics & Critical Value & Remark $($ Alpha $=\mathbf{5 \% )}$ \\
\hline Length & 0.096 & 0.04 & Reject normality \\
Waist & 0.142 & 0.04 & Reject normality \\
Thigh & 0.185 & 0.04 & Reject normality \\
Hip & 0.207 & 0.04 & Reject normality \\
Bottom_girth & 0.349 & 0.04 & Reject normality \\
\hline
\end{tabular}
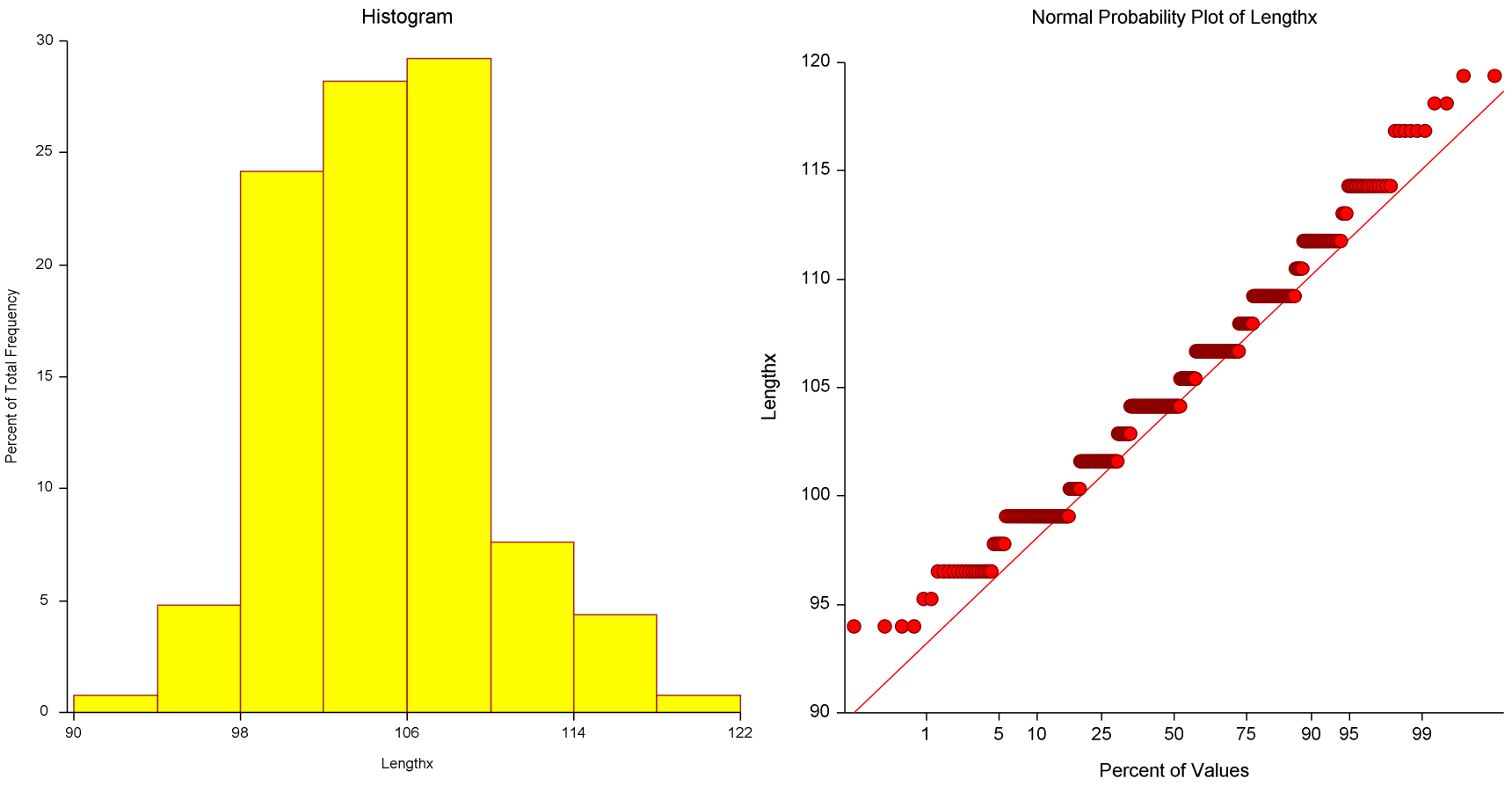

Figure 1. Plots Section of Length. 

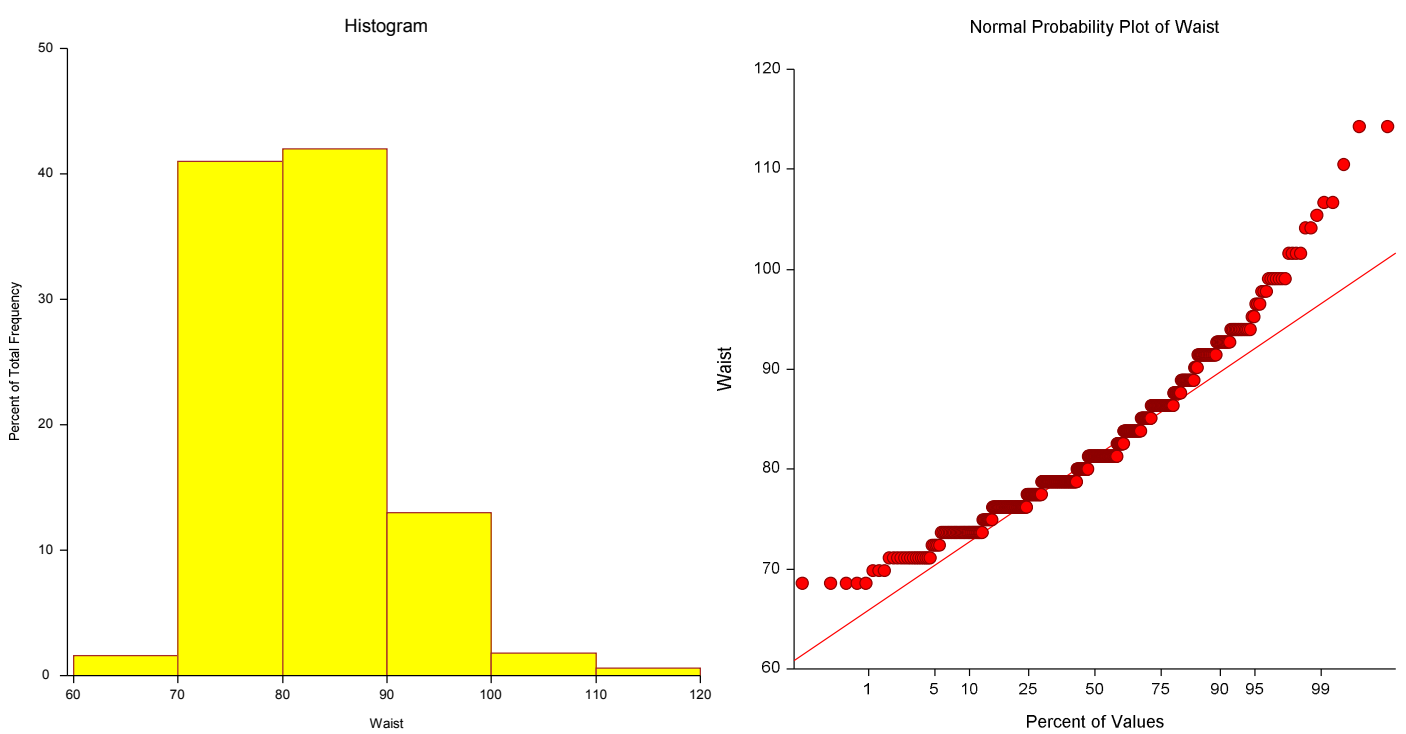

Figure 2. Plots Section of Waist.
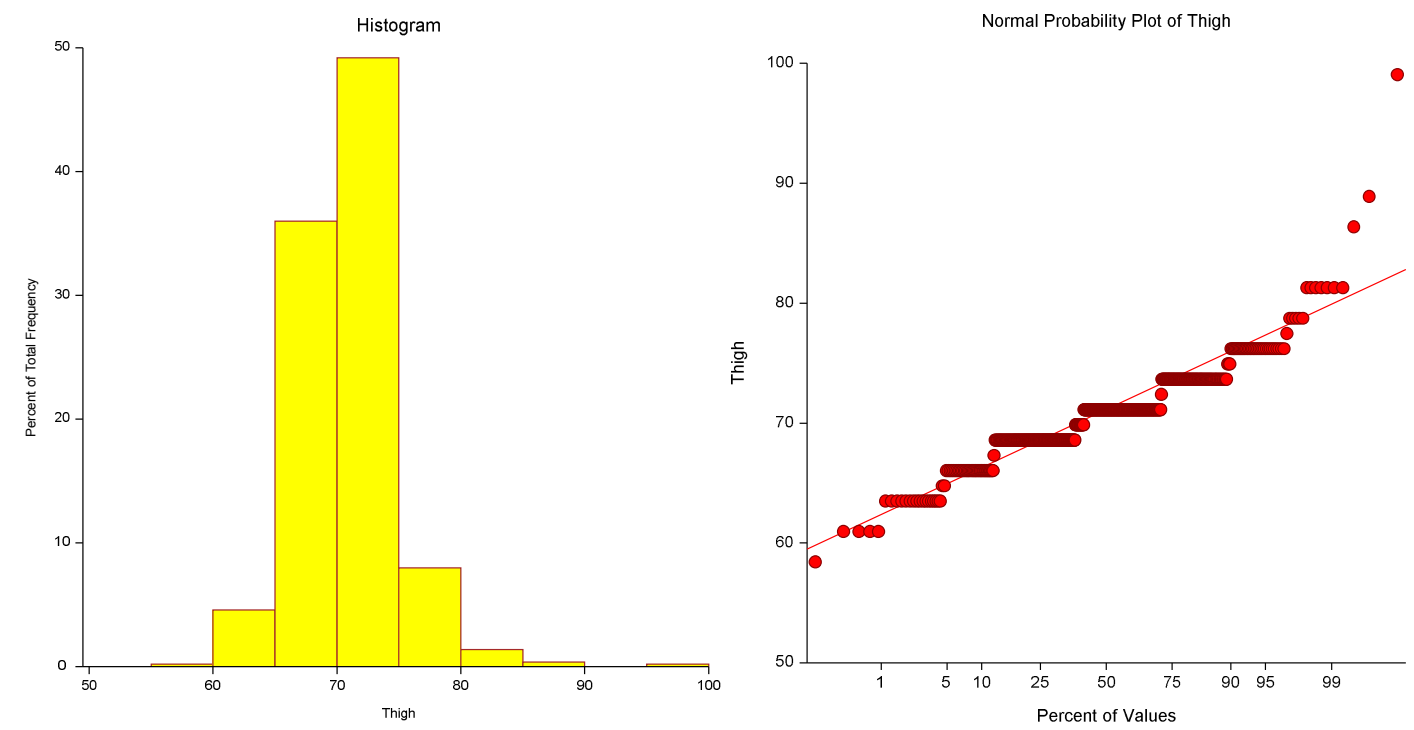

Figure 3. Plots Section of Thigh
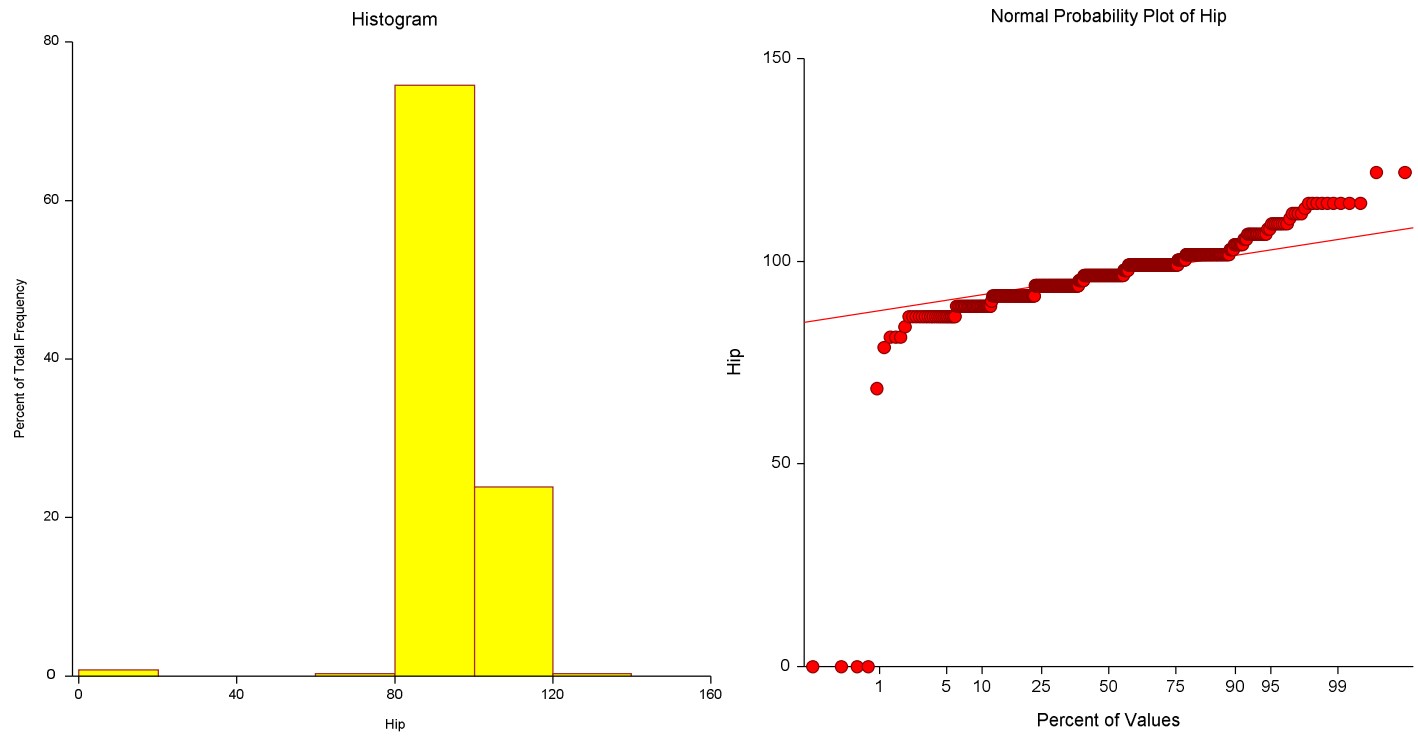

Figure 4. Plots Section of Hip. 

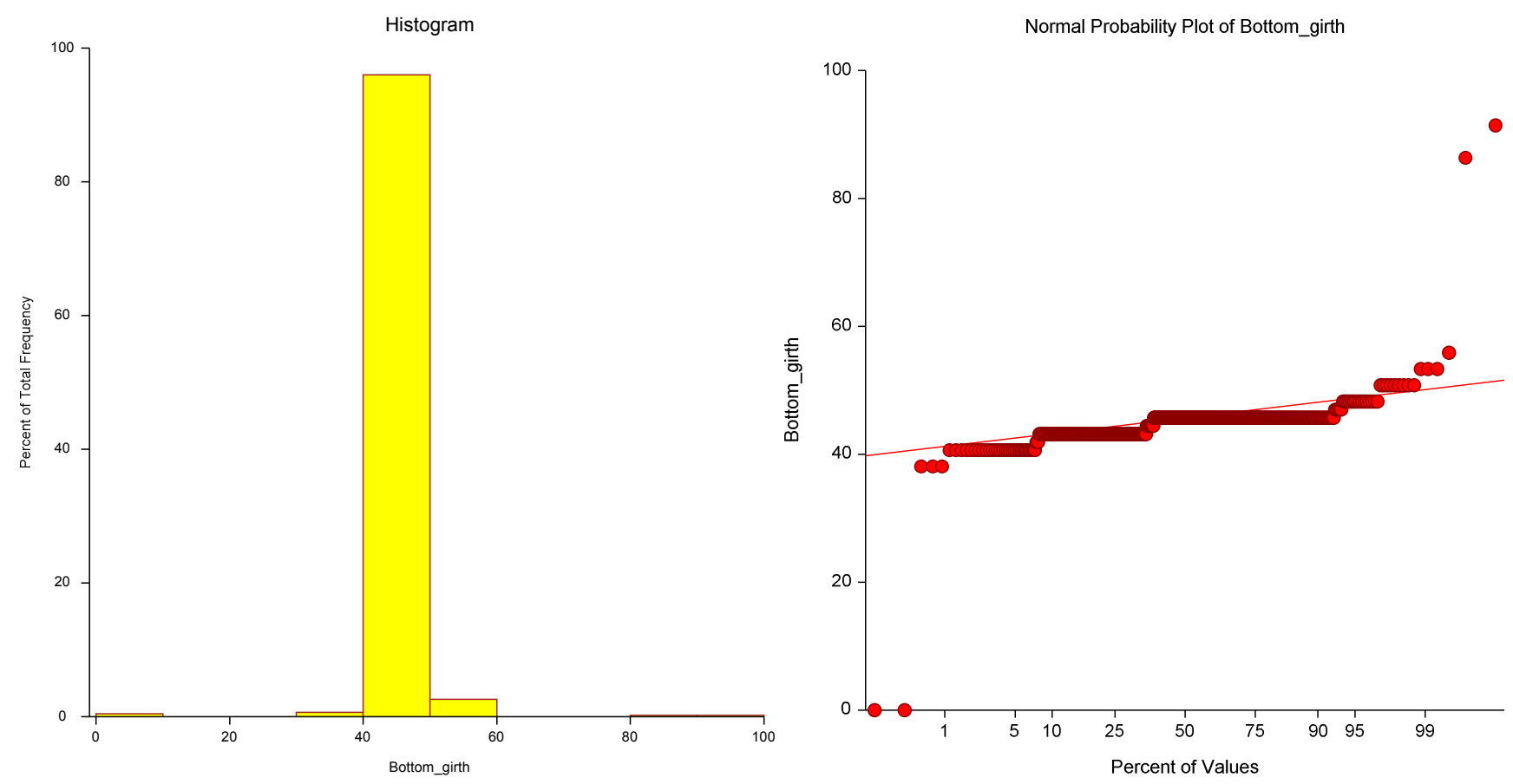

Figure 5. Plots Section of Bottom_girth.

Table 3. Cluster Center per Attribute (Dimensions in cm)

\begin{tabular}{llllll}
\hline \multirow{2}{*}{ Cluster } & Attribute & & & \\
\cline { 2 - 6 } & Length & Waist & Thigh & Hip & Bottom \\
\hline S & 102.2 & 76.2 & 67.9 & 90.2 & 44.1 \\
M & 104.0 & 80.5 & 69.9 & 94.3 & 44.6 \\
L & 104.8 & 78.4 & 71.3 & 97.1 & 44.9 \\
XL & 105.6 & 86.4 & 71.6 & 98.3 & 45.4 \\
XXL & 107.2 & 93.8 & 74.2 & 104.3 & 45.6 \\
\hline
\end{tabular}

Table 4. Fit Values per cluster.

\begin{tabular}{|c|c|c|c|c|c|c|}
\hline Cluster & Rating Scale & Length & Waist & Thigh & Hip & Bottom \\
\hline \multirow[t]{3}{*}{1} & $<0.4$ & 52 & 17 & 31 & 25 & 1 \\
\hline & $0.4-0.6$ & 21 & 20 & 39 & 60 & 0 \\
\hline & $>0.6$ & 25 & 61 & 28 & 13 & 97 \\
\hline No. Of misfit & & 6 & 1 & 4 & 2 & 1 \\
\hline \multirow[t]{3}{*}{2} & $<0.4$ & 21 & 8 & 2 & 12 & 2 \\
\hline & $0.4-0.6$ & 21 & 14 & 7 & 15 & 5 \\
\hline & $>0.6$ & 28 & 48 & 61 & 43 & 63 \\
\hline No. Of misfit & & 1 & 2 & 1 & 2 & 1 \\
\hline \multirow[t]{3}{*}{3} & $<0.4$ & 15 & 54 & 57 & 12 & 8 \\
\hline & $0.4-0.6$ & 55 & 36 & 40 & 36 & 52 \\
\hline & $>0.6$ & 37 & 17 & 10 & 59 & 47 \\
\hline No. Of misfit & & 1 & 3 & 6 & 3 & 2 \\
\hline \multirow[t]{3}{*}{4} & $<0.4$ & 19 & 61 & 14 & 68 & 1 \\
\hline & $0.4-0.6$ & 52 & 51 & 35 & 31 & 0 \\
\hline & $>0.6$ & 56 & 15 & 78 & 28 & 126 \\
\hline No. Of misfit & & 2 & 4 & 1 & 1 & 1 \\
\hline \multirow[t]{3}{*}{5} & $<0.4$ & 10 & 18 & 53 & 73 & 7 \\
\hline & $0.4-0.6$ & 29 & 35 & 25 & 20 & 32 \\
\hline & $>0.6$ & 59 & 45 & 20 & 5 & 59 \\
\hline No. of misfit & & 1 & 2 & 2 & 1 & 3 \\
\hline
\end{tabular}




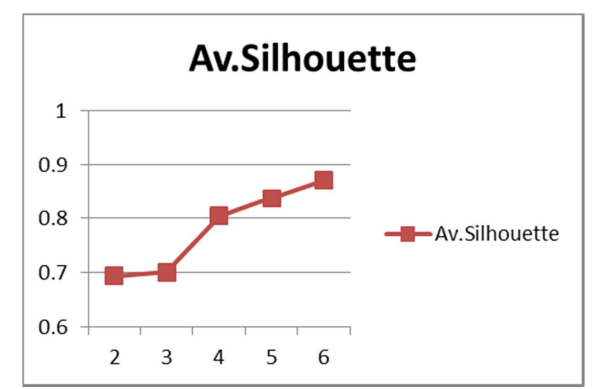

Figure 6 Average Silhouette Vs Number of Cluster.

\section{Conclusion and Recommendation}

Garment fit may be described as a multi-criterion problem as both explicit and implicit aspect of customer's requirements must be taken into consideration for garment design. Such implicit requirements are hidden and not clearly understood through the use of body measurements. They may be associated with the dress culture, style and preference at an instance but are expected to be satisfied by the designer. This work focuses on how to develop size charts which reflect subjectivity in garment selection as a function of measured fit, styles and customer preferences at different instances. A Fuzzy C-Means clustering methodology was introduced as clustering model which account for subjectivity in data mining. The size chart developed for male trouser of various types and styles can also be used for joint replenishment policy by retailers as well as production plan for manufacturers.

However, to improve accuracy of the size chart across clusters and measurement attributes, other clustering techniques may be used. A complete size chart may also be developed for a complete formal and native male wear and more data set obtained for such analysis.

\section{Appendix}

Table A1. AnthropometricData For Male Adults (Trousers).

\begin{tabular}{|c|c|c|c|c|c|}
\hline Customer & Length (cm) & Waist (cm) & Thigh (cm) & Hip (cm) & Bottom girth (cm) \\
\hline 1. & 107.95 & 76.2 & 71.12 & 101.6 & 45.72 \\
\hline 2. & 99.06 & 86.36 & 73.66 & 105.41 & 44.45 \\
\hline 3. & 105.41 & 83.82 & 73.66 & 100.33 & 44.45 \\
\hline 4. & 106.68 & 83.82 & 71.12 & 97.79 & 45.72 \\
\hline 5. & 106.68 & 78.74 & 73.66 & 96.52 & 45.72 \\
\hline 6. & 106.68 & 78.74 & 68.58 & 99.06 & 45.72 \\
\hline 7. & 116.84 & 86.36 & 73.66 & 102.87 & 45.72 \\
\hline 8. & 114.3 & 101.6 & 76.2 & 110.49 & 45.72 \\
\hline 9. & 104.14 & 78.74 & 69.85 & 97.79 & 44.45 \\
\hline 10. & 105.41 & 80.01 & 69.85 & 100.33 & 45.72 \\
\hline 11. & 109.22 & 80.01 & 71.12 & 100.33 & 45.72 \\
\hline 12. & 101.6 & 77.47 & 73.66 & 97.79 & 45.72 \\
\hline 13. & 104.14 & 93.98 & 73.66 & 107.95 & 45.72 \\
\hline 14. & 110.49 & 81.28 & 69.85 & 96.52 & 45.72 \\
\hline 15. & 105.41 & 81.28 & 71.12 & 96.52 & 45.72 \\
\hline 16. & 102.87 & 76.2 & 69.85 & 95.25 & 45.72 \\
\hline 17. & 99.06 & 85.09 & 71.12 & 95.25 & 45.72 \\
\hline 18. & 110.49 & 78.74 & 71.12 & 96.52 & 45.72 \\
\hline 19. & 99.06 & 74.93 & 68.58 & 93.98 & 44.45 \\
\hline 20. & 100.33 & 76.2 & 68.58 & 95.25 & 43.18 \\
\hline 21. & 106.68 & 81.28 & 69.85 & 97.79 & 45.72 \\
\hline 22. & 104.14 & 74.93 & 68.58 & 93.98 & 45.72 \\
\hline 23. & 99.06 & 87.63 & 71.12 & 93.98 & 45.72 \\
\hline 24. & 109.22 & 71.12 & 69.85 & 95.25 & 45.72 \\
\hline 25. & 116.84 & 81.28 & 69.85 & 100.33 & 45.72 \\
\hline 26. & 104.14 & 78.74 & 68.58 & 96.52 & 45.72 \\
\hline 27. & 107.95 & 81.28 & 72.39 & 97.79 & 46.99 \\
\hline 28. & 107.95 & 78.74 & 71.12 & 100.33 & 45.72 \\
\hline 29. & 100.33 & 73.66 & 69.85 & 96.52 & 43.18 \\
\hline 30. & 104.14 & 82.55 & 71.12 & 99.06 & 45.72 \\
\hline 31. & 102.87 & 72.39 & 68.58 & 95.25 & 44.45 \\
\hline 32. & 110.49 & 77.47 & 71.12 & 101.6 & 45.72 \\
\hline 33. & 101.6 & 73.66 & 69.85 & 95.25 & 44.45 \\
\hline 34. & 104.14 & 85.09 & 69.85 & 97.79 & 45.72 \\
\hline 35. & 101.6 & 83.82 & 69.85 & 93.98 & 45.72 \\
\hline 36. & 104.14 & 83.82 & 73.66 & 102.87 & 45.72 \\
\hline 37. & 101.6 & 77.47 & 69.85 & 100.33 & 45.72 \\
\hline 38. & 109.22 & 78.74 & 71.12 & 100.33 & 45.72 \\
\hline 39. & 114.3 & 86.36 & 73.66 & 101.6 & 45.72 \\
\hline
\end{tabular}




\begin{tabular}{|c|c|c|c|c|c|}
\hline Customer & Length (cm) & Waist (cm) & Thigh (cm) & Hip (cm) & Bottom girth $(\mathrm{cm})$ \\
\hline 40. & 105.41 & 78.74 & 68.58 & 101.6 & 45.72 \\
\hline 41. & 111.76 & 78.74 & 69.85 & 100.33 & 45.72 \\
\hline 42. & 105.41 & 81.28 & 71.12 & 100.33 & 45.72 \\
\hline 43. & 111.76 & 78.74 & 69.85 & 97.79 & 45.72 \\
\hline
\end{tabular}

\section{References}

[1] Izzah N, Rahman A, Dawal SZ, Yusoff N, Sofia N, Kamil M. Anthropometric measurements among four Asian countries in designing sitting and standing workstations. Sādhanā [Internet]. 2018; 43 (1): 1-9. Available from: https://doi.org/10.1007/s12046-017-0768-8

[2] Otieno, A. D., Mehtre, A., Fera O, Lema O., O., Gebeyehu S. Developing Standard Size Charts for Ethiopian Men between the Ages of 18-26 through Anthropometric Survey. J Text Apparel, Technol Manag. 2016; 10 (1): 1-10.

[3] Hsu C, Lee T, Kuo H. Mining the Body Features to Develop Sizing Systems to Improve Business Logistics and Marketing Using Fuzzy Clustering Data Mining. WSEAS Trans Comput. 2009; 8 (7): 1215-24.

[4] Adelaja O, Salusso CJ. Designing apparel for Nigerian women : addressing visual appeal, body type and sizing. In: International Textile and Apparel Association Annual Conference Proceedings. 2015. p. 1-3.

[5] Salusso. A method for classifying adult female body form variation in relation to the US Standard for apparel sizing. Doctoral Dissertation, University of Minnesota; 1982.

[6] Tryfos P. An integer programming approach to the apparel sizing problem. J Oper Res Soc. 1986; 37 (10): 1001-6.

[7] McCulloch, C. E., Paal, B. and Ashdown SP. An optimization approach to apparel sizing. J Oper Res Soc. 1998; 49 (5): $492-$ 9.

[8] Gupta, D. and Gangadhar P. A statistical model for developing body size charts for garments. Int J Cloth Sci Technol. 2004; 16: 458-69.

[9] Gupta, Deepti, N. Garg KA and NP. Developing Body Measurement Charts for Garment Manufacture Based on a Linear Programming Approach. J Text Apparel, Technol Manag. 2006; 5 (1): 1-13.

[10] S. Z. Loker, S. Ashdown KS. Size-specific analysis of body scan data to improve apparel fit, J. of Textile and Apparel. J Text Apparel, Technol Manag. 2005; 4 (3): 1-13.

[11] Lee Y-S. Standards Sizing for Clothing based on Anthropometry Data. J Ergon Soc Korea. 2014; 33 (5): 337 54.

[12] Beshah B, Belay B, Tilahun S, Tizazu B, Matebu A. Anthropometric data of Bahirdar City' $s$ adult men for clothing design. Int J Vocat Tech Educ. 2014; 6 (5): 51-7.

[13] J SS and, Pandya S. An Overview of Partitioning Algorithms in Clustering Techniques. Int J Adv Res Comput Eng Technol. 2016; 5 (6): 1943-6.
[14] Zakaria, N., Mohd, J. S., Taib, N., Tan, Y. Y. and Wah YB. Using data mining technique to explore anthropometric data towards the development of sizing system. Int Symp Inform Technol. 2008; 2: 1-7.

[15] Bagherzadeh, R., Latifi, M. and Faramarzi AR. Employing a three-stage data mining procedure to develop sizing system. WASJ. 2010; 8: 923-929.

[16] Elfaki EF, Ali AHM. A Comparison Between The New Established Sizing Systems Sud And Sur Military Clothing Factory Sizing Chart For Poshirt (U4)-Part 1. Int J Adv Res Eng Appl Sci. 2016; 5 (1): 32-41.

[17] M. Vishnu Vardhana Rao, Sharad Kumar and G. N. V. Brahmam. A study of the geographical clustering of districts in Uttar Pradesh using nutritional anthropometric data of preschool children. Indian J Med Res. 2013; 137 (1): 73-81.

[18] Majumder J, Sharma LK. Identifying Body Size Group Clusters from Anthropometric Body Composition Indicators. J Ecophysiol Occup Hlth. 2015; 15: 81-8.

[19] Gupta, Deepti, Garg, Naveen, Arora Komal PN. Developing body measurement charts for Garment Manufacture Based on a Linear Programming Approach. J Text Apparel, Technol Manag. 2006; 5 (1): 1-13.

[20] Rasheed A, Zeng X, Thomassey S. An Approach to the Design of a Fuzzy Logic Model for the Ease Allowance Calculation in Loose Fitting Knee Length Ladies Trousers. J Eng Fiber Fabr. 2013; 8 (4): 4-9.

[21] Cui L, Zhang Y, Deng J, Xu M. A novel multi-item joint replenishment problem considering multiple type discounts. PLoS One [Internet]. 2018; 13 (6): 1-19. Available from: http://dx.doi.org/10.1371/journal.pone.0194738.

[22] Bezdek J. Pattern Recognition with Fuzzy Objective Function, New York. Plenum,. 1981.

[23] Ghosh S. Comparative Analysis of K-Means and Fuzzy CMeans Algorithms. 2013; 4 (4): 35-9.

[24] Hogo MA. Evaluation of E-Learners Behaviour using Different Fuzzy Clustering Models : A Comparative Study. Int J Comput Sci Inf Secur. 2010; 7 (2): 131-40.

[25] Kolawole A. Investigation of The Relationship between Fit and Garment Sizing Parameters. University of Ibadan, Nigeria; 2016.

[26] Dallal G Wilkinson L. An Analytic Approximation to the Distribution of Lilliefors's Test Statistic for Normality. Am Stat. 1986; 40 (4): 294-6.

[27] Hernández N, Mattila H, Berglin L. A systematic model for improving theoretical garment fit. J Fash Mark Manag An Int J. 2018; 22 (4): 527-39. 